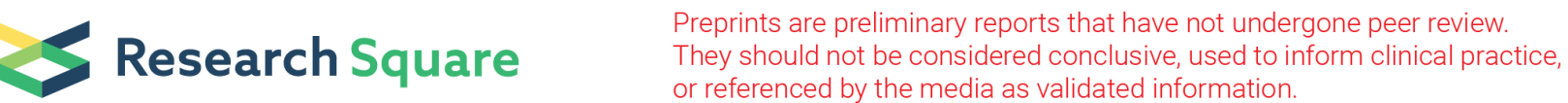

\section{Genetic Characterization and Recombinant History of a Novel Emerging HIV-1 Second-Generation Circulating Recombinant Form (CRF120_0107) Identified Among Sexually Transmitted Infections in Shenzhen, China}

\section{Bo Zhu}

Anhui Medical University

Jin Zhao

Shenzhen Center for Disease Control and Prevention

Xiaorui Wang

Beijing Institute of Microbiology and Epidemiology

Hanping Li

Beijing Institute of Microbiology and Epidemiology

Yongjian Liu

Beijing Institute of Microbiology and Epidemiology

Chenli Zheng

Shenzhen Center for Disease Control and Prevention

Lei Jia

Beijing Institute of Microbiology and Epidemiology

Tianyi Li

Beijing Institute of Microbiology and Epidemiology

\section{Xiaolin Wang}

Beijing Institute of Microbiology and Epidemiology

\section{Lin Chen}

Shenzhen Center for Disease Control and Prevention

Jingyun Li

Beijing Institute of Microbiology and Epidemiology

\section{Bohan Zhang}

Beijing Institute of Microbiology and Epidemiology

Jingwan Han

Beijing Institute of Microbiology and Epidemiology

\section{Lin Li ( $\nabla$ dearwood@sina.com )}

Beijing Institute of Microbiology and Epidemiology 


\section{Research Article}

Keywords: HIV, CRFs, MSM, SGR, NFLG

Posted Date: December 13th, 2021

DOI: https://doi.org/10.21203/rs.3.rs-1147274/v1

License: (c) (i) This work is licensed under a Creative Commons Attribution 4.0 International License. Read Full License 


\section{Abstract}

Under the background of the main epidemic HIV strains (CRF01_AE and CRF07_BC) co-circulation in China, more HIV second-generation recombinant (SGR) strains with CRF01_AE and CRF07_BC as the backbone are emerging. In this study, we analyzed the characteristics and evolutionary history of a newly emerging HIV-1 CRF120_0107 composed of CRF01_AE and CRF07_BC based on the near full-length genome (NFLG) in Shenzhen, Guangdong Province, China. NFLG phylogenetic analysis revealed that these sequences formed a distinct monophyletic branch with a high bootstrap value ( $>90 \%)$, distantly related to all known HIV-1 genotypes. Recombination analysis showed that CRF120_0107 was composed of the predominant HIV-1 strains in China: CRF01_AE and CRF07_BC. Further subregional phylogenetic

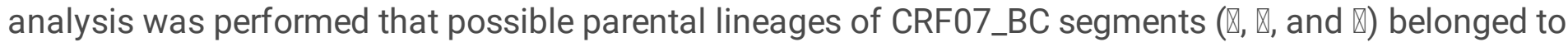
the CRF07_BC men who have sex with men cluster (MSM cluster), other CRF01_AE segments also mainly belonged to MSM Cluster (such as CRF01_AE Cluster 5). Bayesian analysis results inferred that CRF120_0107 placed its emergence in Shenzhen approximately between 2009-2011. The appearance of CRF120_0107 further highlights that more and more HIV-1 SGR strains containing CRF01_AE and CRF07_BC will be more generated frequently and will most likely be more conducive to accelerating the spread of HIV in China. This highlighted it is necessary to monitor MSM high-risk individuals with HIV-1 CRF01_AE and CRF07_BC dual infection to prevent the generation of CRF01_AE/CRF07_BC recombinant strains, thus reducing the possibility of HIV-1 genotype resistance and the complexity of treatment in China.

\section{Background}

The co-circulation of multi-strains of the human immunodeficiency virus (HIV) facilitates the generation of various inter-genotype unique recombinant forms (URFs) and novel circulating recombination forms (CRFs)[1] which will have an important impact on HIV genetics and evolution. The main characteristics of HIV are extremely high genetic variation and high-speed replication ability. Up to now, 118 CRFs and abundant URFs have been reported worldwide constantly and all of them have been included in the Los Alamos National Laboratory (LANL) HIV Sequence Database (http://www.hiv.lanl.gov/). Similarly, in China, the distribution of HIV-1 genotypes has changed dramatically over time, and especially two CRFs, CRF01_AE and CRF07_BC, have led to a national or regional HIV-1 pandemic in recent years[2].

Shenzhen, as one of the four central cities in the Guangdong-Hong Kong-Macao Greater Bay Area (GBA), is located along the coast of Guangdong Province in South China, where the epidemic trend of HIV-1 has become increasingly complicated. It has been found that since 2010, the most prevalent strain among men who have sex with men (MSM) infected with HIV-1, CRF01_AE has been replaced by CRF07_ BC in Shenzhen[3]. In addition, novel HIV-1 second-generation recombinant (SGR) strains with CRF01_AE or CRF07_BC as the backbone have also been discovered in Shenzhen[4], suggesting that CRF01_AE and CRF07_BC are undergoing higher frequency recombination during the HIV-1 epidemic, and adaptive mutation and evolution occurred constantly. In this study, we analyzed the characteristics and 
evolutionary history of a newly emerging HIV-1 CRF120_0107 composed of CRF01_AE and CRF07_BC in Shenzhen, Guangdong Province, China.

\section{Methods}

Three patients (Patient ID: LS14873, LS15083, and LS16846) infected with the novel HIV-1 recombinant strains were included in this study. All participants signed written informed consent. They were infected through sexual transmission in Shenzhen, China. All of them were males with ages between 31 and 38 years old. There was no direct epidemiological linkage among these individuals. The demographic and epidemiological data of these patients are shown in Table 1.

Table 1

Demographic characteristics and epidemiological data of study subjects.

\begin{tabular}{|llllllll|}
\hline $\begin{array}{l}\text { Patient } \\
\text { ID }\end{array}$ & $\begin{array}{l}\text { Sampling } \\
\text { year }\end{array}$ & Region & Gender & Age & $\begin{array}{l}\text { Marital } \\
\text { status }\end{array}$ & $\begin{array}{l}\text { Risk } \\
\text { factor }\end{array}$ & $\begin{array}{l}\text { Accession } \\
\text { no. }\end{array}$ \\
\hline LS15083 & 2015 & SZ, GD & Male & 31 & unmarried & MSM & OK662598 \\
\hline LS16846 & 2015 & SZ, GD & Male & 32 & married & SH & OK662596 \\
\hline LS14873 & 2015 & SZ, GD & Male & 38 & unmarried & MSM & OK662597 \\
\hline $13 S J 011$ & 2013 & SJ, HE & Male & UK & UK & SH & KX198574 \\
\hline $\begin{array}{l}\text { *SZ: Shenzhen city; GD: Guangdong province; SJ: Shijiazhuang city; HE: Hebei province; UK: } \\
\text { Unknown; MSM: men who have sex with men; SH: Heterosexual. }\end{array}$ \\
\hline
\end{tabular}

To obtain the near full-length genome (NFLG) sequences of the three patients in Shenzhen, viral RNAs were extracted from plasma samples and then reverse transcribed to cDNA. Two halves genomic segments with 1000bp overlapping regions were amplified by nested polymerase chain reaction (PCR) with Platinum $^{\mathrm{TM}}$ Taq DNA Polymerase High Fidelity (Invitrogen) as described before[5] and sequenced. All sequence fragments of each sample were assembled by using the ContigExpress software. Sequence quality analysis was carried out by the LANL HIV Sequence Database tool: quality control. Finally, two NFLG sequences about 8800 bp (LS14873: 8877bp, HXB2: 762-9613nt; LS16846: 8894bp, HXB2: 7629613nt) and a 5'-half genome sequence (LS15083: 4271bp, HXB2: 790-5096nt) were obtained. These sequences were submitted to GenBank and the accession numbers are OK662596-OK662598.

In order to search for the sequences with the highest similarity (> 95\%), HIV Basic Local Alignment Search Tool (BLAST) was performed using all sequences obtained in this study as the query set. Another patient (Patient ID: 13SJ011) was a heterosexual (SH) male in Shijiazhuang of Hebei province, China, the sequence named 13SJ011 (2630bp, HXB2:790-3455nt, access number: KX198574) was obtained and exhibited a high degree of genetic similarity with three strains in this study. Then, these 4 sequences were further aligned with HIV-1 reference sequences (major pure subtypes, CRFs, and HIV-1 O Group) by Multiple Alignment using Fast Fourier Transform (MAFFT) online service[6]. NFLGs maximum-likelihood (ML) phylogenetic tree was produced by PhyML 3.0 (maximum-likelihood phylogeny) under the General 
Time Reversible (GTR) and Gamma distributed with Invariant sites $(\mathrm{G}+\mathrm{l})$ model with approximate Shimodaira-Hasegawa-like likelihood ratio test (aLRT SH-like) values[7].

To demonstrate possible unique inter-subtype recombinant structures, the jumping profile hidden Markov model (jpHMM) (http://jphmm.gobics.de/submission_hiv)[8] and Similarity Plotting (SimPlot v3.5.1)[9] were employed to perform recombination analysis. Recombinant pattern of the novel recombinant genome was generated by Recombinant HIV-1 Drawing Tool. Subregion phylogenetic tree was constructed by the neighbor-joining method based on the Kimura 2-parameter model with 1000 bootstrap replicates in Molecular Evolutionary Genetics Analysis (MEGA6) to verify the origins of these subregions[10]. The program TempEst v1.5.1 (TEMPoral Exploration of Sequences and Trees) was performed to evaluate the sampling time signal for data firstly (R-square $>0.7$ )[11]. Next, the time of the most recent common ancestor (TMRCA) of recombinant segments was conducted by Bayesian Evolutionary Analysis Sampling Trees (BEAST v1.10)[12] with a GTR+G+I nucleotide substitution model, an uncorrelated lognormal relaxed clock model, a Bayesian Skyline tree prior, $2 \otimes 10^{8}$ length of chain sampling frequency of 1000. All parameters' effective sample size (ESS) was greater than 200. All phylogenetic trees were visualized by Interactive Tree Of Life (iTOL) online [13].

\section{Results And Discussion}

Phylogenetic analysis of these 4 sequences showed that they did not cluster with any known HIV-1 subtypes/CRFs and formed an independent cluster with high bootstrap value ( $>90 \%)$, indicating that they may belong to a novel genotype (Fig. 1A). And then both the BLAST and recombinant analysis revealed that these strains comprised of HIV-1 CRF01_AE and CRF07_BC segments may be a new CRF with 5 identical breakpoints: 3 CRF01_AE and 3 CRF07_BC segments (Fig. 1B).

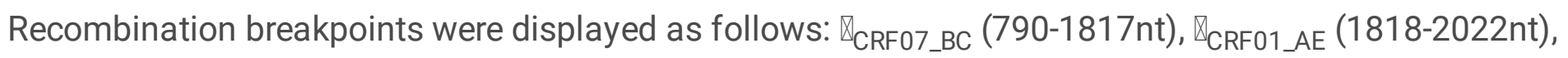

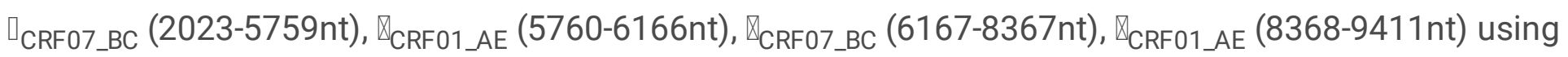
HXB2 as a reference (Fig. 1C). As shown in Fig. 2, further subregional phylogenetic analysis results

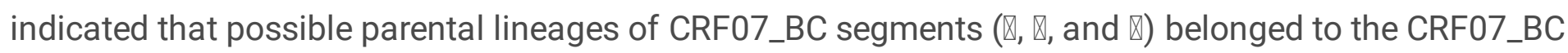

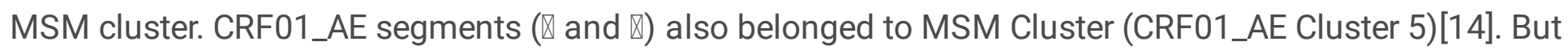
segment $\triangle$ may be too short or insufficient gene similarity, only 13SJ011 sample was located in CRF01_AE Cluster4 (MSM Cluster), other sequences form a cluster by themselves among CRF01_AE Cluster. Subregional phylogenetic analysis revealed that all recombinant segments are closely related to the clusters associated with the sexually transmitted population, especially the MSM population.

Bayesian analysis was performed to estimate the time to the most recent common ancestor (tMRCA).

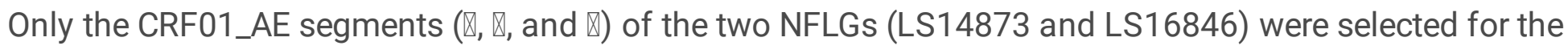
next time-evolution analysis, because the only CRF01_AE segment ( () the other two non-NFLG sequences (13SJ011 and LS15083) contain is too short to be detected sufficient time signal by the TempEst

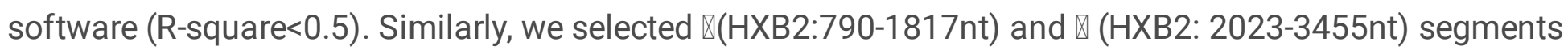
of CRF07_BC recombination segments to do the Bayesian analysis, for the reasons that all sequences 
include the 2 segments and the sequence set of these 2 segments reached enough time signals (R-square $>0.7$ ). Maximum clade credibility (MCC) trees were reconstructed based on the recombinant regions of

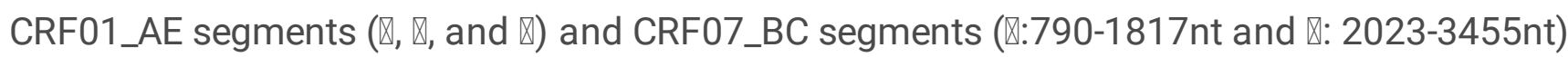
respectively. CRF01_AE and CRF07_BC sequences with sampling years were both downloaded from the LANL HIV Sequence Database and obtained from the HIV-infected blood samples collected by our laboratory. All these sequences were subjected to the phylodynamics analysis together with the target sequences in this study. As shown in Fig. 3, the tMRCA of novel recombinant strains for CRF01_AE

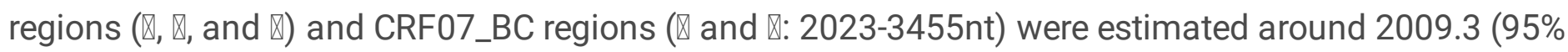
highest probability density (HPD): 2007.0-2011.2) and 2011.7 (95\%HPD: 2010.8-2012.5), respectively. However, a sequence (Patient ID: 13SJ011) in the CRF07_BC region was estimated to be around 2008.2 (95\%HPD: 2007.4-2009.0) and not clustered with other three sequences obtained in Shenzhen, which may be the earlier ancestor of the three Shenzhen sequences, or it may be of distinct origin but sharing the same recombination breakpoints with others. The sequence is too short for us to know for sure. Since all patients have no direct epidemiological relationship and the strains that they infected share the same mosaic structure, according to the HIV-1 CRF nomenclature proposal[15], three recombinant strains in Shenzhen are recognized and included by the HIV database and designated as the new CRF (CRF120_0107). Together with the fact that CRF120_0107 has not previously been described in other countries/regions, the results inferred that CRF120_0107 placed its emergence in Shenzhen approximately between 2009-2011 (Fig. 3).

With the rapid economic development and large population flow base, Shenzhen has great potential for the spread of HIV locally and even across the country. The origin and outbreak of CRF55_01B among MSM in Shenzhen has changed HIV-1 molecular epidemiology patterns in the region, and perhaps across the nation[16, 17]. In addition, since the discovery of CRF01_AE/CRF07_BC recombinants, the MSM population has contributed to the emergence of most URFs_0107 and CRFs_0107 Second-generation CRFs like CRF120_0107 have been identified especially among the MSM population, which has become the special high-risk group of the form and prevalence of the recombinants. At present, it is obvious that the advantages of these strains in the generation and spread among MSM in the region may be the accumulation of power for its high transmission in the future. At the same time, the generation of HIV-1 second-generation recombinant forms (CRF01_AE/CRF07_BC) also acts as an evolutionary force to increase HIV diversity and complexity in this region.

\section{Conclusion}

In this study, we firstly identified and analyzed the characteristics of the newly emerging HIV-1 CRF120_0107 composed of CRF01_AE and CRF07_BC in Shenzhen, China, and analyzed its evolutionary history. The emergence of CRF120_0107 indicated that more and more strains with CRF01_AE and CRF07_BC as parents form SGR strains, suggesting that CRF01_AE/CRF07_BC SGR strains may have become the dominant strains in China. In view of the prevalence of most URFs_0107 and CRFs_0107 in MSM population, we emphasize that MSM high-risk individuals with CRF01_AE and CRF07_BC dual infection should be closely monitored and treated in advance to prevent the generation of 
CRF01_AE/CRF07_BC recombinant strain from causing more widespread transmission and epidemic, so as to avoid increasing the possibility of genotype resistance and the complexity of treatment work.

\section{Abbreviations}

aLRT SH-like: approximate Shimodaira-Hasegawa-like likelihood; BEAST: Bayesian Evolutionary Analysis Sampling Trees; BLAST: Basic Local Alignment Search Tool; CRFs: circulating recombination forms; ESS: effective sample size; GBA: Guangdong-Hong Kong-Macao Greater Bay Area; GTR: General Time Reversible; G+I: Gamma distributed with Invariant sites; HIV: human immunodeficiency virus; iTOL: Interactive Tree Of Life; jpHMM: jumping profile hidden Markov model; LANL: Los Alamos National Laboratory; MAFFT: Multiple Alignment using Fast Fourier Transform; MCC: Maximum clade credibility; MSM: men who have sex with men cluster; NFLG: near full-length genome; PCR: polymerase chain reaction; PhyML: maximum-likelihood phylogeny; SimPlot: Similarity Plotting; MEGA: Molecular Evolutionary Genetics Analysis; ML: maximum-likelihood; MSM: men who have sex with men; SGR: second-generation recombinant; SH: heterosexual; TempEst: TEMPoral Exploration of Sequences and Trees; tMRCA: the most recent common ancestor; URFs: unique recombinant forms;

\section{Declarations}

\section{Acknowledgments}

The authors thank all of the participants and peer workers.

\section{Author Contributions}

$\mathrm{BZ}, \mathrm{JH}$ and LL designed the study; BZ and JZ performed the experiments; JZ, CZ and LC collected samples; $X R W$ and $Y L$ and participated in sequences assembly. $H L$ and $L J$ participated in phylogenetic analyses; $B Z$ and $X R$ W participated in recombinant analysis; $T L, X L W, B H Z$ collected the demographic data and HIV reference sequences; $\mathrm{BZ}, \mathrm{XR} \mathrm{W}, \mathrm{JL}, \mathrm{JH}$ and $\mathrm{LL}$ participated in the writing process and manuscript correction. All authors read and approved the final manuscript.

\section{Funding}

This study was supported by the National Key Research and Development Program of China (2020YFA0907000), the NSFC $(81773493,31800149,31900157)$, the State Key Laboratory of Pathogen and Biosecurity (AMMS), the National $13^{\text {th }}$ Five-Year Grand Program on Key Infectious Disease Control (2018ZX10721102, 2018ZX10732101-001-003), Guangdong Basic and Applied Basic Research Foundation (2019B1515120003), San-Ming Project of Medicine in Shenzhen (SZSM201811071).

\section{Availability of data and materials}

The gene sequences of LS16846, LS14873, and LS15083 were deposited in the GenBank with the accession number: OK662596- OK662598. The sequence 13SJ011 was obtained from Los Alamos 
National Laboratory HIV Sequence Database with the accession number: KX198574.

\section{Ethics approval and consent to participate}

Not applicable.

\section{Consent for publication}

Not applicable.

\section{Competing interests}

The authors declare no competing interests.

\section{References}

1. Gao Q, Feng Y, Gao L, Zhang M, Liu Y, Xiao M, Dong X, Xia X: Genetic characteristics of a New HIV-1 subtype B/C intersubtype circulating recombinant form (CRF118_BC) identified in Yunnan, China. $J$ Infect 2021, 83:237-279.

2. Li X, Li W, Zhong P, Fang K, Zhu K, Musa TH, Song Y, Du G, Gao R, Guo Y, et al: Nationwide Trends in Molecular Epidemiology of HIV-1 in China. AIDS Res Hum Retroviruses 2016, 32:851-859.

3. Zhao J, Chen L, Chaillon A, Zheng C, Cai W, Yang Z, Li G, Gan Y, Wang X, Hu Y, et al: The dynamics of the HIV epidemic among men who have sex with men (MSM) from 2005 to 2012 in Shenzhen, China. Sci Rep 2016, 6:28703.

4. Wang X, Zhao J, Li X, Li H, Zhang Y, Liu Y, Chen L, Zheng C, Jia L, Han J, et al: Identification of a novel HIV-1 second-generation Circulating Recombinant Forms CRF109_0107 in China. The Journal of infection 2020.

5. Li L, Liang S, Chen L, Liu W, Li H, Liu Y, Bao Z, Wang Z, Zhuang D, Liu S, Li J: Genetic characterization of 13 subtype CRF01_AE near full-length genomes in Guangxi, China. AIDS Res Hum Retroviruses 2010, 26:699-704.

6. Katoh K, Rozewicki J, Yamada KD: MAFFT online service: multiple sequence alignment, interactive sequence choice and visualization. Brief Bioinform 2019, 20:1160-1166.

7. Guindon S, Dufayard JF, Lefort V, Anisimova M, Hordijk W, Gascuel O: New algorithms and methods to estimate maximum-likelihood phylogenies: assessing the performance of PhyML 3.0. Syst Biol 2010, 59:307-321.

8. Schultz AK, Zhang M, Bulla I, Leitner T, Korber B, Morgenstern B, Stanke M: jpHMM: improving the reliability of recombination prediction in HIV-1. Nucleic Acids Res 2009, 37:W647-651.

9. Zhang C, Ding N, Wei JF: Different sliding window sizes and inappropriate subtype references result in discordant mosaic maps and breakpoint locations of HIV-1 CRFs. Infect Genet Evo/2008, 8:693697. 
10. Tamura K, Stecher G, Peterson D, Filipski A, Kumar S: MEGA6: Molecular Evolutionary Genetics Analysis version 6.0. Mol Biol Evol 2013, 30:2725-2729.

11. Rambaut A, Lam TT, Max Carvalho L, Pybus OG: Exploring the temporal structure of heterochronous sequences using TempEst (formerly Path-O-Gen). Virus evolution 2016, 2:vew007.

12. Suchard MA, Lemey P, Baele G, Ayres DL, Drummond AJ, Rambaut A: Bayesian phylogenetic and phylodynamic data integration using BEAST 1.10. Virus Evol 2018, 4:vey016.

13. Letunic I, Bork P: Interactive Tree Of Life (iTOL) v5: an online tool for phylogenetic tree display and annotation. Nucleic Acids Res 2021, 49:W293-w296.

14. Feng Y, He X, Hsi JH, Li F, Li X, Wang Q, Ruan Y, Xing H, Lam TT, Pybus OG, et al: The rapidly expanding CRF01_AE epidemic in China is driven by multiple lineages of HIV-1 viruses introduced in the 1990s. Aids 2013, 27:1793-1802.

15. Robertson DL, Anderson JP, Bradac JA, Carr JK, Foley B, Funkhouser RK, Gao F, Hahn BH, Kalish ML, Kuiken C, et al: HIV-1 nomenclature proposal. Science (New York, NY) 2000, 288:55-56.

16. Zai J, Liu H, Lu Z, Chaillon A, Smith D, Li Y, Li X: Tracing the transmission dynamics of HIV-1 CRF55_01B. Sci Rep 2020, 10:5098.

17. Zhang D, Zheng C, Li H, Li H, Liu Y, Wang X, Jia L, Chen L, Yang Z, Gan Y, et al: Molecular surveillance of HIV-1 newly diagnosed infections in Shenzhen, China from 2011 to 2018. J Infect 2021, 83:76-83.

\section{Figures}


A

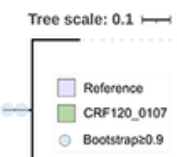

B
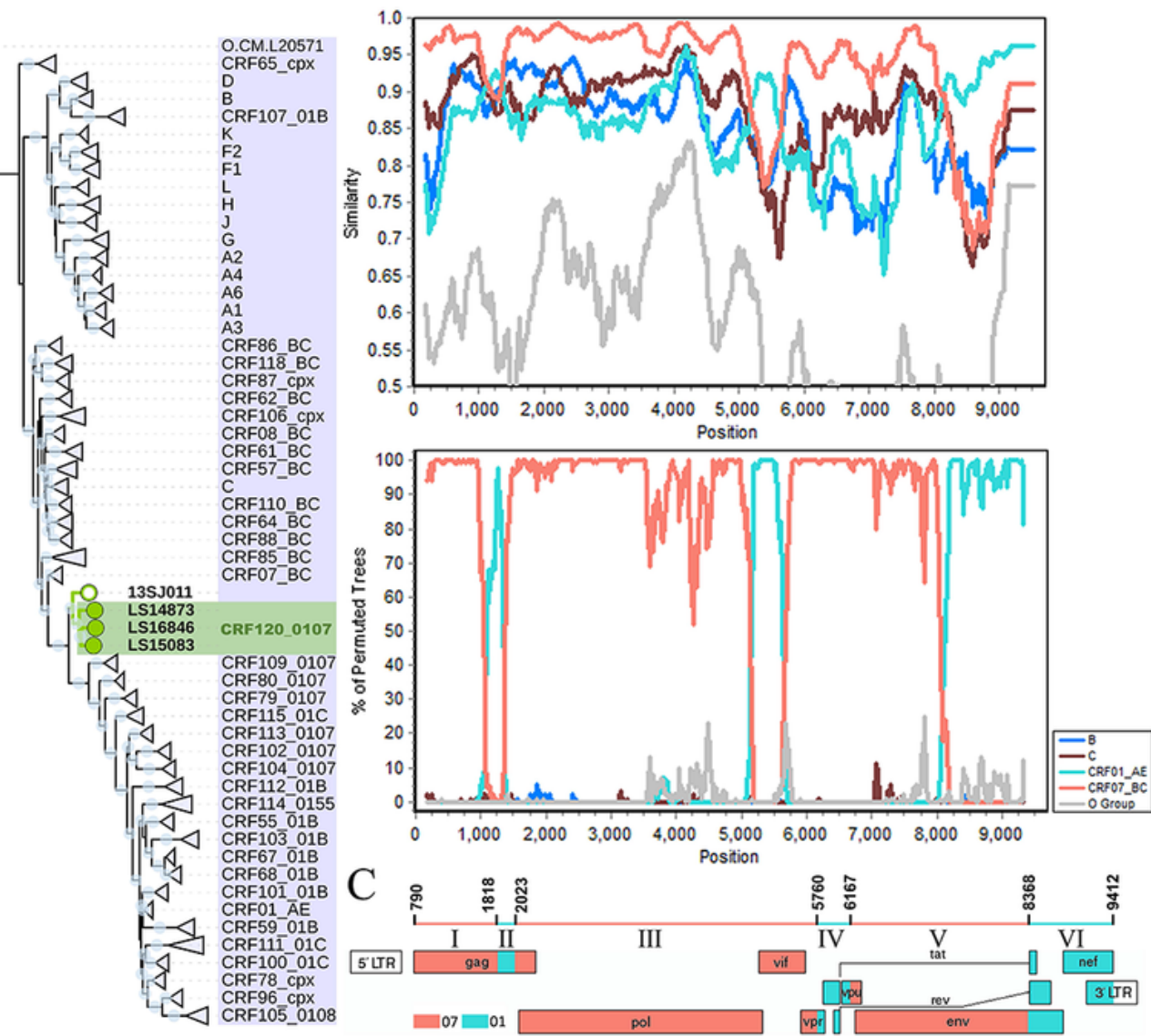

Fig. 1 Maximum-likelihood (ML) phylogenetic analysis based on HIV-1 CRF120_0107 near full-length genomes (NFLGs). (A) CRF120_0107 sequences were marked with a green solid circle (๑), suspected CRF120_0107 sequence(13SJ011) was marked with a hollow green circle $(\odot)$, and the corresponding sequence labels were also marked with a light green background. The ML tree was constructed by phyML3.0 based on the GTR+G+I model with aLRT SH-like values. HIV-1 reference sequences were HIV-1 pure subtypes, main CRFs in China, and HIV-1 O Group obtained from the LANL HIV Sequence Database. Only bootstrap values $\geq 0.9$ are presented at the nodes of the tree marked with a light blue solid circle. (B) Recombinant analysis was performed by Simplot 3.5 .1 with a window size of 350 and a step size of 20 bp to identify parental strains. Subtype B, Subtype C, CRF01_AE, CRF07_BC, and HIV-1 O group were used as references. (C) Recombinant gene structure maps were drawn by $\begin{array}{llll}\text { Recombinant } & \text { HIV-1 } & \text { Drawing } & \text { Tool }\end{array}$

(https://www.hiv.lanl.gov/content/sequence/DRAW CRF/recom mapper.html).

Figure 1

See image above for figure legend 

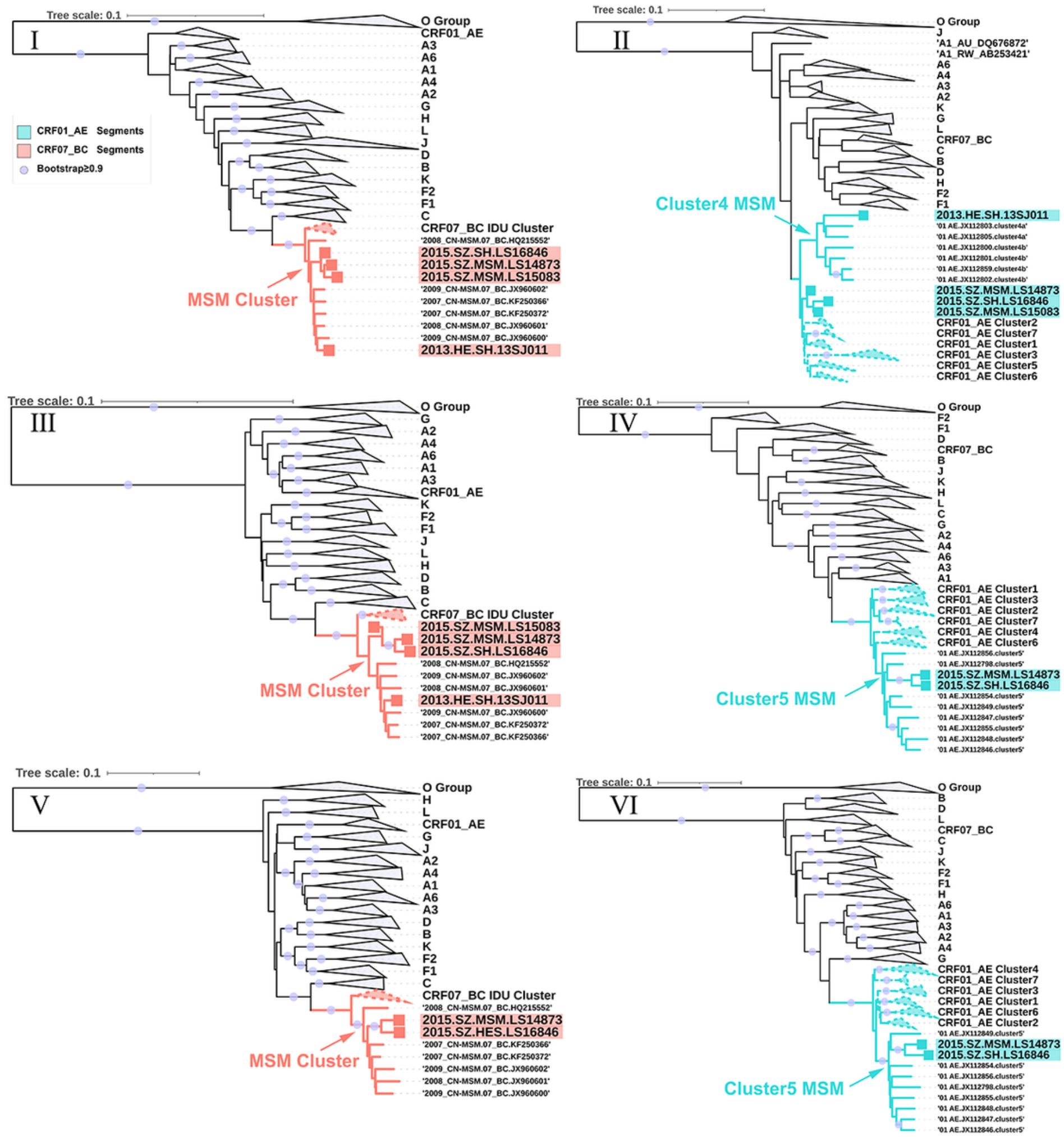

Figure 2

Subregion phylogenetic analysis based on CRF01_AE or CRF07_BC segments of HIV-1 CRF120_0107. Subregion neighbor-joining tree was constructed by the Kimura 2-parameter model of nucleotide

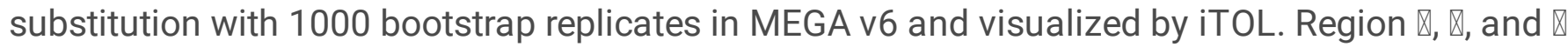

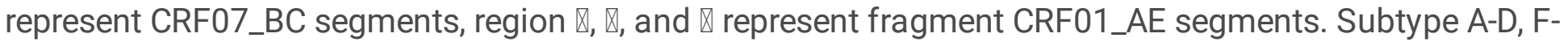
H, J-L, CRF01_AE, CRF07_BC, and HIV-1 O group were used as references. The CRF01_AE or CRF07_BC 
segments sequences of CRF120_0107 and suspected CRF120_0107 sequence (13SJ011) were marked with " $\square$ " and the corresponding sequence labels were also marked with a cyan and pink background respectively. Only bootstrap values $\geq 90 \%$ were presented at the nodes of the tree marked with a light purple solid circle.

CRF07_BC Segments

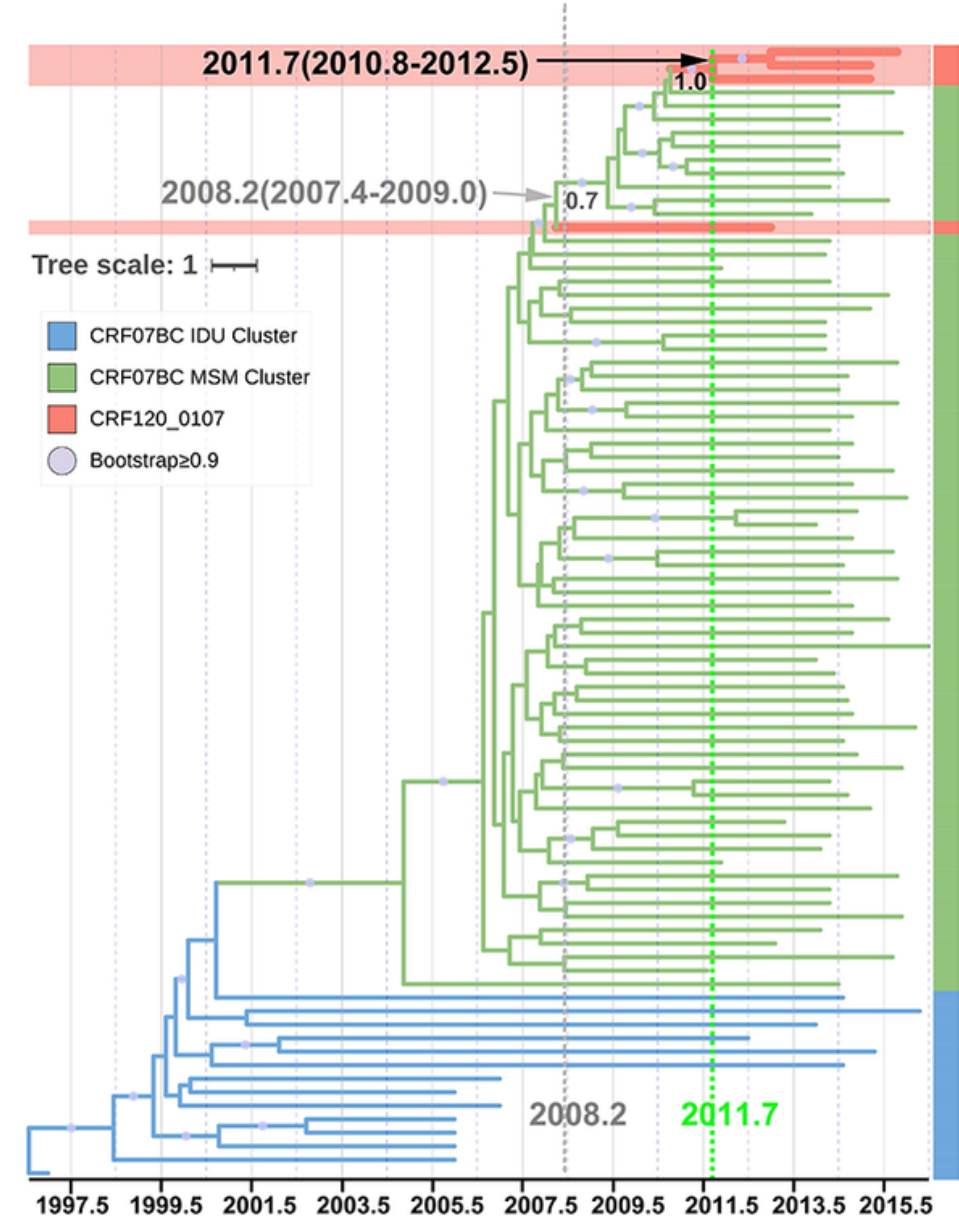

\section{CRF01_AE Segments}

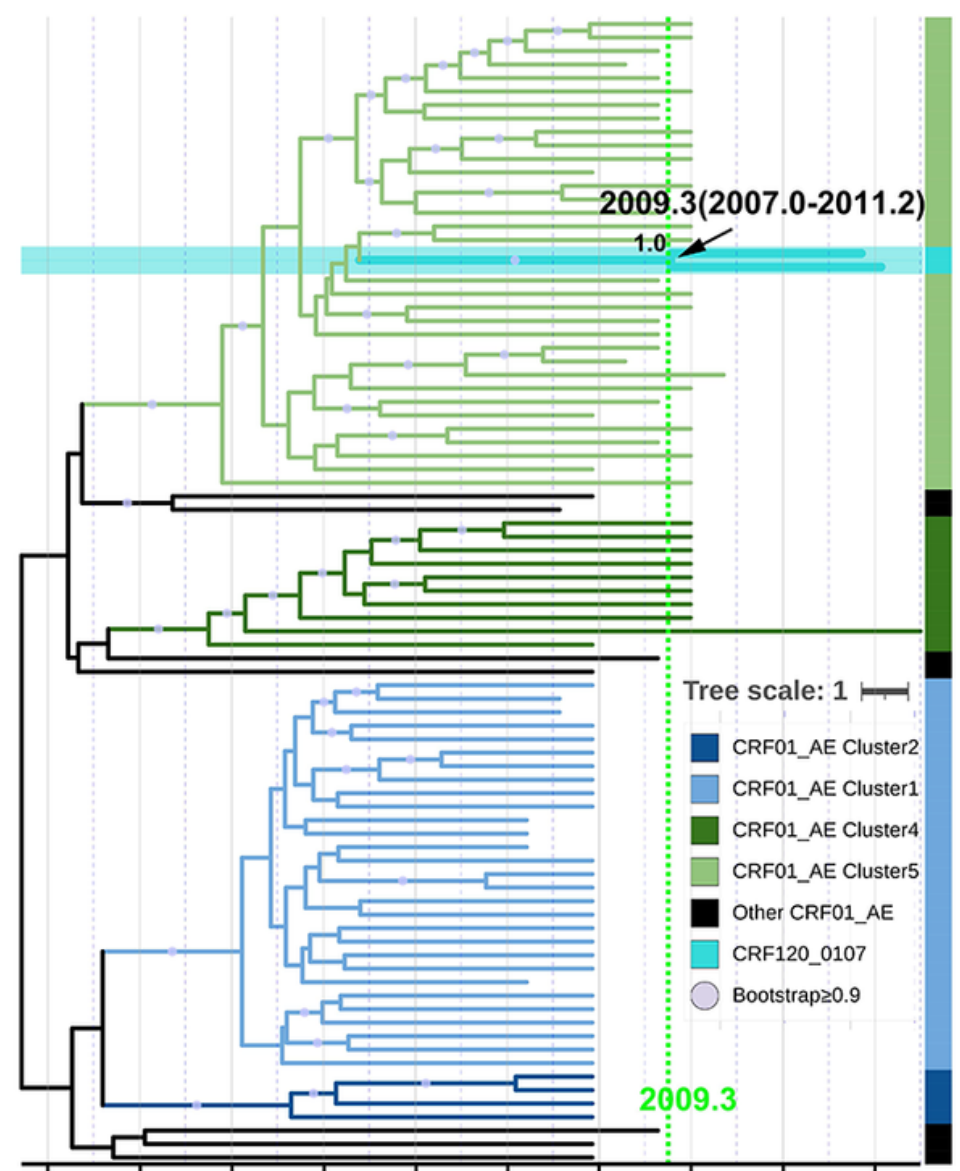

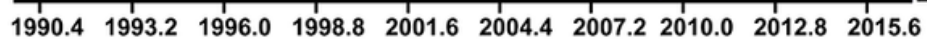

\section{Figure 3}

Evolution analysis based on CRF01_AE or CRF07_BC segments of HIV-1 CRF120_0107. MCC trees show

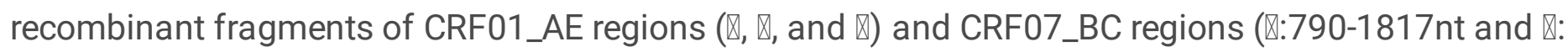
2023-3455nt). Timescale is shown below the tree. The mean tMRCA and $95 \%$ highest probability density (HPD) for the CRF120_0107 and suspected CRF120_0107 sequence (13SJ011) nodes are displayed. CRF120_0107 strains and suspected CRF120_0107 strain (13SJ011) are highlighted cyan for the CRF01_AE region and pink for the CRF07_BC region. 